Interactive comment on "Black carbon modelling in urban areas: investigating the influence of resuspension and non-exhaust emissions in streets using the Street-in-Grid (SinG) model" by Lya Lugon et al.

Lya Lugon et al.

lya.lugon@enpc.fr

Received and published: 27 August 2021

article [utf8]inputenc [a4paper, total=6in, 8in]geometry xurl comment 
Black carbon modelling in urban areas: investigating the influence of resuspension and non-exhaust emissions in streets using the Street-in-Grid (SinG) model :

\section{Answers to referees' comments}

August 27, 2021

\section{Anonymous Referee \#2}

Received and published: 30 July 2021

\subsection{General comments}

This research article provides new and very useful information regarding the contribution of on-road traffic to urban air pollution. The focus is on particulate black carbon (BC) and its vehicular emission sources. The authors use a novel advanced modeling technique, the SinG model, which simulates urban air pollution at various spatial scales 
(urban background and street-level pollution) in an internally consistent manner. Furthermore, they improved this model by implementing a more detailed dry deposition model and conducting a dynamic simulation of the emission, deposition, and resuspension of particulate matter.

In this work, they apply this model to investigate the vehicular emissions of BC. In particular, they evaluate the relative contributions of exhaust and non-exhaust (tire, brake, and road wear) emissions of BC to air pollution in streets. The non-exhaust emission source is particularly uncertain and the authors do an excellent job at reviewing the literature, identifying the major uncertainty sources, and systematically investigating their impact on simulated BC concentrations. They conduct a thorough evaluation of the modeling results using experimental results of a field program that provides both ambient air BC concentrations in a Paris suburban street and deposited PM mass in that same street. This evaluation allows the authors to propose that the greater estimates of non-exhaust emissions are more realistic and that current non-exhaust BC emission inventories may significantly underestimate actual $\mathrm{BC}$ vehicular emissions.

This conclusion is consistent with the fact that some earlier modeling studies needed an arbitrary increase in $\mathrm{BC}$ vehicular emissions to match observations. The fact that their comparisons with both BC air concentrations and deposited mass are consistent is particularly interesting, because it provides closure to the air pollution process being modeled. In addition, this work confirms the importance of treating urban air quality at various scales in a joint consistent manner. Previous work by the authors and others had demonstrated this point for reactive gaseous pollutants. It is demonstrated here for chemically inert particulate matter.

Therefore, I recommend publication of this work in GMD with minor modifications to address the following comments. Also, the text needs some editing by the authors to correct some English mistakes and improve the style overall. 
1. The conclusion that non-exhaust $B C$ emissions are currently underestimated in European emission inventories (according to the results presented here) has important policy implications. It means that replacing internal-combustion engines by electric vehicles will not eliminate $B C$ air pollution, but only reduce it by about half (in terms of vehicular emissions). This point should be highlighted, both in the conclusion and in the abstract. Clearly, additional work (mostly experimental) should be conducted to confirm this result, but the work presented here makes a very strong case for revising current BC vehicular emission inventories and taking non-exhaust emissions explicitly into account in air quality simulations. Some recommendations regarding additional studies to refine those results could be provided in the conclusion.

Author response: Yes, thank you. These points are now highlighted. At lines 19-23, the sentences

"Non-exhaust emission, such as brake and tyre and road wear, largely contribute to BC emissions, with a contribution equivalent to exhaust emissions. Here, emission factors of tyre, brake and road wear are calculated based on the literature, and a sensitivity analysis of these emission factors on BC concentrations in streets is performed. The model to measurement comparison shows that tyre-emission factors usually used in Europe are probably under-estimated, and tyre-emission factors coherent with some studies of the literature and the comparison performed here are proposed."

are replaced by

"Non-exhaust emissions, such as brake and tyre and road wear, may largely contribute to $B C$ emissions, with a contribution equivalent to exhaust emissions. Here, a sensitivity analysis of $B C$ concentrations is performed by comparing simulations with different emission factors of tyre, brake and road wear. The different emission factors considered are estimated based on the literature. We found a satisfying model-to- 
measurement comparison using high tyre-wear emission factors, which may indicate that tyre-emission factors usually used in Europe are probably under-estimated. These results have important policy implications: public policies replacing internal-combustion engines by electric vehicles may not eliminate $B C$ air pollution, but only reduce it by half."

Also, at line 409, the sentence

"They can be as relevant as exhaust emissions, and their underestimation may justify the virtual increase of $B C$ emissions often employed in street-network modelling studies."

is followed by

"This can have direct consequences on public policies aiming at reducing $B C$ concentrations in urban areas: non-exhaust emissions may contribute to as much as half of the $B C$ concentrations in streets.".

Finally, at line 501, the sentence

"Following the literature, increasing the $B C$ passenger cars tyre-wear emission factors of the EMEP guidelines from $1.36 \mathrm{mg} . \mathrm{vkm}^{-1}$ to $20.8 \mathrm{mg} . \mathrm{vkm}^{-1}$ lead to good comparisons of the simulated $B C$ concentrations to the measured ones."

is followed by

"The authors highlight that additional work, mostly experimental, should be conducted to confirm this result. However, the work presented here makes a very strong case for revising current $B C$ vehicular emission inventories and taking non-exhaust emissions explicitly into account in air quality simulations."

2. The introduction is rather long (about one quarter of the entire text) and the authors should consider breaking this current introductory section into two sections: (1) a gen- 
eral introduction and (2) a section that provides an overview of the current state of the science for non-exhaust vehicular emissions (since it corresponds to the main topic of this work).

Author response: As suggested, the Introduction was divided in two sections: Introduction and Uncertainties and variability of emissions factors observed in non-exhaust emissions. The Introduction starts at line 25, until line 64. At line 64, the sentence

"On the other hand, non-exhaust emissions are still not well-known in the literature, and they are not included in regulatory emissions legislation."

is followed by

"Non-exhaust emissions consist of particles emitted from vehicles operation and linked to tyre, brake and road wear and resuspension. Their emission factors are greatly variable and associated to large uncertainties. Uncertainties in non-exhaust emissions can be explained by the difficulty to dissociate emissions from the different processes and by the great variability of: (i) tyre, brake and road constituents used in different locations; (ii) vehicles characteristics, such as weight, location of driving wheels, etc; (iii) vehicles operational conditions, such as vehicles speed and ambient temperature; and (iv) methodologies to determinate wear emission factors, i.e. direct measurements insitu, with wind-tunnel experiments or receptor-oriented methods (Boulter, 2005; Thorpe and Harrison, 2008). These uncertainties are reflected in the estimation of the contribution of non-exhaust emissions to PM concentration observed in the literature, as detailed in Section 2.".

Then, it is followed by lines 177-200.

The new section Uncertainties and variability of emissions factors observed in nonexhaust emissions corresponds to lines 71-176. Please, note that this section starts by the new sentences

"As mentioned in the introduction, non-exhaust emissions still present high uncertain- 
ties, and a great variability of emission factors is observed the literature. This section presents an overview of the different studies that estimate particle non-exhaust emissions. It also includes some studies that investigate their BC content.".

3. In the first paragraph of the introduction, it is stated that BC is mainly emitted by traffic. It should be pointed out that during winter, wood burning (for residential heating) is also an important source of BC in some areas (including the Paris region).

Author response: At line 29, the sentence

"Because BC is mainly emitted by traffic, it is often used as a traffic pollutant tracer (Invernizzi et al., 2011; de Miranda et al., 2019)."

is replaced by

"BC is mainly emitted by traffic, and then it is often used as a traffic pollutant tracer (Invernizzi et al., 2011; de Miranda et al., 2019). During winter the wood burning in residential heating is also an important source of $B C$ in some regions, such as Paris city (AIRPARIF, 2012).".

4. Among the potential health effects of $B C$, should its carcinogenic effects be mentioned (e.g., Lequy et al., Environ. Health Perspectives, 129, March 2021)?

Author response: Yes, thank you. The sentence at line 30

"A special attention is given to BC because of its potential impacts on human health: recent studies showed a strong correlation between $B C$ concentrations and the occurrence of respiratory and cardiovascular problems, others indicated an alteration on fetus development (Jansen et al., 2005; Highwood and Kinnersley, 2006; Janssen et al., 2011; Dons et al., 2012; Zhang et al., 2019a, b)." 
is replaced by

"A special attention is given to $B C$ because of its potential impacts on human health. Recent studies showed a strong correlation between $B C$ concentrations and the occurrence of cancers (Leguy et al., 2021), respiratory and cardiovascular problems (Jansen et al., 2005; Highwood and Kinnersley, 2006; Janssen et al., 2011; Dons et al., 2012), and also impacts on fetus development Zhang et al., 2019a, b).".

5. The sentence on the environmental impacts of $B C$ needs to be rewritten, because visibility reduction is due to the radiative effects of BC. I suggest: "...environmental impacts due to its radiative properties (light absorption), which lead to visibility reduction (references) and global warming (references).

Author response: Yes, thank you. The sentence at line 33

" $B C$ is also responsible for environmental impacts, such as visibility reduction (Tao et al., 2009; Chen et al., 2016; Li et al., 2019) and radiative effects (Jacobson, 2001; Chung and Seinfeld, 2005; Tripathi et al., 2005; Ramachandran and Kedia, 2010)."

is replaced by

" $B C$ is also responsible for environmental impacts due to its radiative properties (light absorption), which lead to visibility reduction (Tao et al., 2009; Chen et al., 2016; Li et al., 2019) and global warming (Jacobson, 2001; Chung and Seinfeld, 2005; Tripathi et al., 2005; Ramachandran and Kedia, 2010)".

6. p. 2, line 49: delete "in models" (the under-estimation affects the emission inventory in general, which may be used for modeling or other tasks, such as reporting).

Author response: The sentence 
"The corrections applied to BC emissions in these studies suggest an under-estimation of $B C$ emissions in models."

is replaced by

"The corrections applied to BC emissions in these studies suggest an under-estimation of $B C$ emissions.".

7. p. 3, line 82 and others: the unit mg.vkm-1 is used throughout (milligrams per vehicle-kilometer travelled); however, later in the text (e.g., p. 9, line 255) the unit veh.h-1 (vehicles per hour) is used for traffic flow. Unit notations should be consistent for "vehicles". I suggest using mg.(veh-km)-1 for the former.

Author response: It was corrected.

8. On p. 4, lines 107-109, it is mentioned that the road-wear emissions calculated by Thouron et al. (2018) were greater than those of the EMEP guidelines. Can the authors specify whether this is due to the algorithm used or the road characteristics (or a combination of both)?

Author response: It is due to the combination of both, algorithm and road characteristics (represented by the $h_{\text {pavement }}$ ).

At lines 107-109, the sentence

"However, the road-wear emissions calculated using the NORTRIP model with the soil characteristics employed by Thouron et al. (2018) in "Boulevard Alsace Lorraine" (a street East of Paris) are higher than those proposed in the EMEP guidelines, by a ratio 6.0."

is replaced by 
"The road-wear emissions calculated using the NORTRIP model with the road characteristics employed by Thouron et al. (2018) in "Boulevard Alsace Lorraine" (a street East of Paris). They are higher than those proposed in the EMEP guidelines, by a ratio 6.0 , because of their different algorithm and road characteristics.".

9. p. 4, line 105: I think that "soil" would only apply to a dirt road. I suggest "road characteristics" instead (also p. 13, line 360).

Author response: Yes, thank you. At line 105, the sentence

"Road-wear emissions also take into account the soil characteristics, as the pavement hardness"

is replaced by

"Road-wear emissions also take into account the road characteristics, as the pavement hardness".

Also, at line 360 , the sentence

"Note that for road-wear emissions, the soil characteristics used in Bouvelard Alsace Lorraine by Thouron et al. (2018) were employed, leading to higher road-wear emissions than in the EMEP guidelines."

is replaced by

"Note that for road-wear emissions, the road characteristics used in Bouvelard Alsace Lorraine by Thouron et al. (2018) were employed, leading to higher road-wear emissions than in the EMEP guidelines.".

10. p. 5, line 133: "worst quality codes"; I am not sure what the authors mean. 
In Sections 2 and 3, it is mentioned that particulate BC is treated using a sectional size distribution with 6 size sections, which is useful to correctly simulate dry deposition. Since atmospheric chemistry is not treated, I assume that aerosol dynamics (condensation/evaporation, nucleation, and coagulation) is also not treated. This seems appropriate as it would have little effect on the BC size distribution (in particular, considering the uncertainties associated with its initial size distribution). The authors should mention this point.

Also, the initial size distribution of emitted particulate BC should be specified, either with a reference or by providing the size distribution.

Author response: The "worst quality codes" mentioned in this study refer to the emission factor quality code indicated in European emission guidelines (Ntziachristos and Samaras, 2016). The quality codes indicated for non-exhaust emissions range from $B$ (emission factors non statistically significant based on a small set of measured reevaluated data), C (emission factors estimated on the basis of available literature), to D (emission factors estimated applying similarity considerations and/or extrapolation). To improve clarity, at line 132 the sentences

"Road emission factors vary from $3.8 \mathrm{mg} . v \mathrm{~km}-1$ (Boulter, 2005) to $200 \mathrm{mg} . \mathrm{vkm}-1$ (Thouron et al., 2018), and they present the worst quality codes compared to other wear emission factor. The EMEP guidelines (Ntziachristos and Boulter, 2016) quantify the typical error associated to road wear emission factors to be between $50 \%$ to $300 \%$, associated to the difficulties to separate precisely the non-exhaust emission sources and the dependence of soil properties and vehicles operational conditions."

are replaced by

"Road emission factors vary from $3.8 \mathrm{mg} . v \mathrm{~km}-1$ (Boulter, 2005) to $200 \mathrm{mg} . \mathrm{vkm}-1$ (Thouron et al., 2018). Compared to other non-exhaust emission sources, road-wear emission factors present the worst quality codes, according to the European emission guidelines EMEP (Ntziachristos and Boulter, 2016), where different quality codes are 
defined for non-exhaust emissions, ranging from $B$ (emission factors non statistically significant based on a small set of measured re-evaluated data), $C$ (emission factors estimated on the basis of available literature), to $D$ (emission factors estimated applying similarity considerations and/or extrapolation). Road wear is the emission source with the worst quality code, and they highlight the difficulties to separate precisely the nonexhaust emission sources and the dependence of road characteristics and vehicles operational conditions.".

Also, at line 210, the sentence

"Because $B C$ is an inert species, this study does not take into account chemical reactions, and only $B C$ concentrations are modelled."

is followed by

"The aerosol dynamics (nucleation, coagulation and condensation/evaporation) is also neglected, as it would have no effect on BC mass concentration and low effect on size distribution.".

The size distribution of non-exhaust particle emissions is specified following the reference of each test. In simulations 2, 4, 5 and 6 followed the size distribution indicated in the European emission guidelines (Ntziachristos and Boulter, 2016). The simulation 3 followed the indications present in the NORTRIP model (Denby et al., 2013a).

These details about the size distribution of emitted particles are included in Section 4.1 (The simulations). At line 355, the sentence

"Simulations 2 employs the BC wear emission factors indicated in the EMEP guidelines, also used in the HERMES model (Guevara et al., 2019; Ntziachristos and Boulter, 2016)."

is replaced by

"Simulation 2 employs the BC wear emission factors and size distribution indicated in 
the EMEP guidelines, also used in the HERMES model (Guevara et al., 2020; Ntziachristos and Boulter, 2016)."

Also, at line 358, the sentence

"Simulation 3 uses the $P M_{10}$ wear emission factors indicated in the NORTRIP model (Denby et al., 2013a)."

is replaced by

"Simulation 3 uses the $P M_{10}$ wear emission factors and size distribution indicated in the NORTRIP model (Denby et al., 2013a)."

And finally, at line 363, the sentence

"Simulation 4 employs the same brake and road-wear emission factors as in the EMEP guidelines, but tyre-wear emission factors are higher."

is followed by

"The same size distribution of non-exhaust emissions indicated in the EMEP guidelines (Ntziachristos and Boulter, 2016) is employed.".

To improve clarity, an additional Table is included in the Appendix section, with some more details of the size distribution of emitted particles from non-exhaust emissions.

Please, note that the sentence "More details about particle size distribution of nonexhaust emissions are indicated in Table A2, in the Appendix A3." is included at line 367.

11. p. 10 , line 280: it is not clear why $Q_{\text {emis,exh }}$ represents both exhaust and nonexhaust emissions. Please clarify.

Author response: It was corrected. 
At line 280 the sentence

"with $Q_{\text {emis,exh }}$ the exhaust and non-exhaust traffic emission rates"

is replaced by

"with $Q_{\text {emis,exh }}$ the exhaust traffic emission rate".

12. p. 10, line 280: it is not clear in $\mathrm{Q}_{d e p, v}$ what the second subscript, $v$, represents.

Please clarify.

Author response: The term $v$ indicates the street volume. This is not exactly the same considered in Equation 7 because, in addition to particle deposition over the street surface, it also takes into account the particle deposition over the building surfaces.

To improve clarity, the sentence

$Q_{d e p, v}$ the deposition flux over the street volume, considering the street pavement and building walls surfaces.

is replaced by

$Q_{\text {dep }, v}$ the deposition flux over the street volume $v$, considering the street pavement and building walls surfaces.

13. In Section 3, p. 12, Equation 10: please specify the units of EF.

Author response: It was added.

14. In Section 4.1 , p. 13, line 343: "to estimate and control"; please specify what "control" refers to. 
Author response: Some studies propose specific measures to control non-exhaust emissions, such as reducing the limit speed in streets and/or smoothing traffic flow (Begi et al, 2020). We decided to remove this word from the introduction, and it seems more coherent to mention the non-exhaust emission control in the conclusion, and the control was not discussed in the introduction. Then, at line 343, the sentence

"As mentioned in the introduction, non-exhaust emissions are difficult to estimate and control."

is replaced by

"As mentioned in the introduction, non-exhaust emissions are difficult to estimate.".

And, at line 504, the sentence

"Following the literature, increasing the BC passenger cars tyre-wear emission factors of the EMEP guidelines from $1.36 \mathrm{mg} . \mathrm{vkm}-1$ to $20.8 \mathrm{mg} . \mathrm{vkm}-1$ lead to good comparisons of the simulated $B C$ concentrations to the measured ones."

is followed by

"Also, more studies are needed to control these emissions. Some studies already indicate the importance of controlling vehicle speed and/or smoothing traffic flow to reduce non-exhaust emissions (Begi et al, 2020). But other aspects can be investigated, such as the road, tyre and brake characteristics, vehicle weight, etc.".

15. At the end of Section 4.1, the authors mention that their correction factor for exhaust $\mathrm{BC}$ emissions used in simulation 6 is based on traffic flow characteristics and BC/PM2.5 uncertainties. Could this be explained in greater detail?

Author response: To improve clarity, the sentences

"Finally, in order to evaluate the influence of uncertainties in the BC speciation of ex- 
haust emissions, simulation 6 uses the same non-exhaust emission factors as simulation 4, but BC exhaust emission factors are artificially increased by $23 \%$. This correction factor is defined based on the traffic-flow characteristics observed in the "Boulevard Alsace Lorraine" during the TRAFIPOLLU campaign, and the BC/PM ${ }_{2.5}$ uncertainties for each vehicle class detailed in Table 3-91 of Ntziachristos and Samaras (2018)."

are replaced by

"Finally, in order to evaluate the influence of uncertainties in the BC speciation of exhaust emissions, simulation 6 uses the same non-exhaust emission factors as simulation 4, but $B C$ exhaust emissions are artificially increased by $23 \%$. This correction factor is defined using a linear correlation based on (i) the traffic-flow characteristics observed in the "Boulevard Alsace Lorraine" during the TRAFIPOLLU campaign (percentage of diesel and petrol vehicles, according to the vehicle category and technology), and (ii) the BC/PM $M_{2.5}$ uncertainties for each vehicle class detailed in Table 3-91 of Ntziachristos and Samaras (2018)."

16. In Section 4.2, the authors provide two different sets of criteria for model performance evaluation. The stricter set includes 6 performance metrics $(5$ were initially defined by Hanna et al., Atmos. Environ., 38, 4675-4687, 2004; the normalized absolute difference was added later), whereas the less strict set (suitable for urban areas according to Hanna and Chang, 2012) includes only 4. It is not clear whether the authors consider that the stricter criteria defined for MG and VG should also apply to the less strict set or whether those metrics must simply be dropped from the less strict set; this point should be clarified. It may be a moot point since, in any case, simulations 1 through 3 fail the less strict criteria (except NMSE) and simulations 4 through 6 meet all the stricter criteria.

Author response: In the less strict criteria the MG and VG are not considered. To improve clarity, the sentence 
"Two different criteria are defined, a most strict criteria, with $-0.3<F B<0.3 ; 0.7<$ $M G<1.3 ; N M S E<3 ; V G<1.6 ; F A C 2 \geq 0.5 ; N A D<0.3$, and a less strict criteria acceptable in urban areas, with $-0.67<F B<0.67$; NMSE $<6$; FAC2 $\geq 0.3 ; N A D<$ 0.5."

is replaced by

"Two different criteria are defined, (i) a lest strict criteria, accepted in urban areas, with $-0.67<F B<0.67$; NMSE $<6$; FAC2 $\geq 0.3 ; N A D<0.5$, and (ii) a most strict criteria, with $-0.3<F B<0.3 ; 0.7<M G<1.3 ; N M S E<3$; VG $<1.6 ;$ FAC2 $\geq 0.5 ; N A D<$ 0.3.".

Also, the sentences

"The BC concentrations observed at "Boulevard Alsace Lorraine" are strongly underestimated in simulations 1, 2 and 3, with a fractional bias (FB) equal to -1.26, -1.10 and -1.15 respectively, not satisfying any performance criterion. The configuration used in simulation 4, with higher tyre and brake-wear emissions, results in satisfactory statistical indicators, respecting all the most strict performance criteria proposed by Hanna and Chang (2012) and Herring and Huq (2018)."

are replaced by

"The BC concentrations observed at "Boulevard Alsace Lorraine" are strongly underestimated in simulations 1, 2 and 3, with a fractional bias (FB) equal to -1.26, -1.10 and -1.15 respectively. They do not satisfy any performance criterion, nor the less strict ones, except for normalised mean square error (NMSE). However, the configurations used in simulations 4, 5 and 6, with higher tyre-wear emissions, result in satisfactory statistical indicators. They respect both the less and most strict performance criteria proposed by Hanna and Chang (2012) and Herring and Huq (2018).".

17. In Section 4.3, p. 17, lines 425-426: I would not say that the observations of Amato C17 
et al. are "quite similar" because the result obtained in this work is slightly above the upper bound of the range given by Amato et al. In Section 4.3, the authors refer to $\mathrm{PM}_{10}$ as fine particles. This definition of $\mathrm{PM}_{10}$ is unfortunately commonly used in France by some agencies, although it is incorrect. Fine particles correspond to $\mathrm{PM}_{2.5}$, and $\mathrm{PM}_{10}$, therefore, includes fine particles and a fraction of coarse particles.

Author response: Yes, thank you, the use of the term fine particles was corrected. The sentence

"Therefore, the average mass density of fine particles $\left(P M_{10}\right)$ in "Boulevard Alsace Lorraine" is about 250 mg.m-2."

is replaced by

"Therefore, the average mass density of $P M_{10}$ in "Boulevard Alsace Lorraine" is about 250 mg.m-2.".

However, both studies (this study and Amato et al.) are focused on $\mathrm{PM}_{10}$. Maybe it was not clear before, but please, see the answer of Minor comment 10, that contains the precision about size distribution.

18. In Section 5, p. 19, line 477: "lower concentrations"? Should it be "higher concentrations" if there is double counting?

Author response: Yes, it was corrected, thank you.

The sentence

"These large differences and the lower concentrations simulated in MUNICH compared to SinG may be explained by the double counting of traffic emissions performed by MUNICH with the one-way coupling technique."

is replaced by 
"These large differences and the lower concentrations simulated in SinG compared to MUNICH may be explained by the double counting of traffic emissions performed by MUNICH with the one-way coupling technique.".

19. As mentioned above, the authors should mention the policy implications of their results concerning $\mathrm{BC}$ emissions from vehicles in the conclusion.

Author response: As indicated in the specific comment 1, at line 409 the sentence

"They can be as relevant as exhaust emissions, and their underestimation may justify the virtual increase of BC emissions often employed in street-network modelling studies."

is followed by

"This can have direct consequences in public policy to strongly reduce $B C$ concentrations in urban areas: non-exhaust emissions may contribute to as much as half of the $B C$ concentrations in streets.".

20. Also, some suggestions for additional experimental studies to confirm the results of their work would be appropriate.

Author response: As indicated in the specific comment 1, at line 501 the sentence

"Following the literature, increasing the $B C$ passenger cars tyre-wear emission factors of the EMEP guidelines from $1.36 \mathrm{mg} . \mathrm{vkm}^{-1}$ to $20.8 \mathrm{mg} \cdot \mathrm{vkm}^{-1}$ lead to good comparisons of the simulated BC concentrations to the measured ones."

is followed by

"The authors highlight that additional work, mostly experimental, should be conducted to confirm this result. However, the work presented here makes a very strong case for 
revising current $B C$ vehicular emission inventories and taking non-exhaust emissions explicitly into account in air quality simulations."

Interactive

comment 